\title{
Qualidade do sono e sonolência excessiva entre estudantes de medicina
}

\author{
Quality of sleep and excessive drowsiness among medical students
}

\author{
Rodrigo Rufino Pereira Silva ${ }^{1}$, Thaise de Abreu Brasileiro Sarmento ${ }^{2}$. \\ Ankilma do Nascimento Andrade Feitosa ${ }^{3}$, Luciana Modesto de Brito ${ }^{4}$
}

Silva RRP, Sarmento TAB, Feitosa ANA, Brito LM. Qualidade do sono e sonolência excessiva entre estudantes de medicina / Quality of sleep and excessive sleepiness among medical students. Rev Med (São Paulo). 2020 jul.-ago.;99(4):350-6.

\begin{abstract}
RESUMO: Introdução: A demanda acadêmica a que são submetidos os alunos do curso de graduação em Medicina tende a alterar sua qualidade do sono, em virtude principalmente da carga horária elevada do curso. A partir do entendimento da importância do sono e de seu impacto na qualidade de vida do estudante é que ressalta-se a notabilidade dessa mensuração. Objetivo: Avaliar a percepção que o estudante de medicina tem acerca da qualidade do próprio sono, bem como o grau de sonolência diurna excessiva, comparando as diferentes fases do curso. Métodos: Trata-se de um estudo transversal e analítico envolvendo 234 acadêmicos do $1^{\circ}$ ao $6^{\circ}$ ano do curso de graduação em Medicina de uma faculdade do alto sertão paraibano. Utilizou-se para mensuração o Índice de Qualidade do sono de Pittsburgh e a Escala de Sonolência de Epworth, ambos questionários validados para uso no Brasil. Resultados: A média global de horas de sono por noite foi de 6,92 horas. Dos 234 entrevistados, $64,5 \%(\mathrm{n}=$ 149) apresentaram qualidade ruim de sono ou distúrbio deste e pelo menos $21 \%$ relataram fazer uso de medicação para dormir. Viu-se ainda que a maior parte da amostra apontou que dorme 7 horas ou menos por noite $(58,6 \%)$. A análise dos dados obtidos pela Escala de Sonolência de Epworth demonstrou que 55,55\% $(\mathrm{n}=130)$ dos estudantes tinham sonolência diurna excessiva que deve ser investigada. Conclusão: Foi observado que a maioria dos graduandos em Medicina apresentou privação de sono associada consequentemente à sonolência excessiva e à qualidade ruim de sono, indicando necessidade de ações preventivas neste sentido. Os resultados apontam, nesse caso, para a pertinência do investimento em esforços para a correção dessa tendência. Fazem-se necessárias medidas de promoção de saúde entre a população de estudantes do ensino superior.
\end{abstract}

Descritores: Transtornos do sono-vigília; Estudantes de medicina; Sonolência; Distúrbios do sono por sonolência excessiva.

\begin{abstract}
Introduction: The demands of the academic life of medical students can alter their quality of life, mainly due to the heavy course load. Understanding the importance of sleep and its impact on the students lives' quality, we emphasize the importance of measuring this parameter. Objective: To evaluate the perception of medical students on the quality of their own sleep and the degree of excessive daytime sleepiness, comparing the different stages of the course. Method: This is a cross-sectional and analytical study with 234 students from the $1^{\text {st }}$ to the $6^{\text {th }}$ year of the undergraduate medical course of a university in the region of the alto sertão paraibano. The Pittsburgh Sleep Quality Index and the Epworth Sleepiness Scale were used for measurement. Both questionnaires are validated for use in Brazil. Results: The mean sleep duration in the overall sample was 6.92 hours. Of the 234 respondents, $64.5 \%(n=149)$ had poor sleep quality or a sleep disorder and at least $21 \%$ reported using sleeping pills. Most of the participants reported a sleep duration of 7 hours or less $(58.6 \%)$. The analysis of the data obtained by the Epworth Sleepiness Scale showed that $55.55 \%(n=130)$ of the students had excessive daytime sleepiness that should be investigated. Conclusion: It was observed that most medical students had sleep deprivation, which was associated with excessive sleepiness and poor sleep quality, indicating the need for preventive actions in this regard. The results point to the relevance of investing in efforts to correct this trend. It is necessary to implement health promotion measures for higher education students.
\end{abstract}

Keywords: Sleep wake disorders; Students, medical; Sleepiness; Disorders of excessive somnolence.

Trabalho realizado com alunos do curso de Graduação em Medicina da Faculdade Santa Maria - FSM - Cajazeiras, PB - Brasil.

1. Estudante do Curso de Graduação em Medicina da Faculdade Santa Maria - FSM - Cajazeiras, PB. https://orcid.org/0000-0003-0428-0305. E-mail: rd ro@hotmail.com.

2. Professora do Departamento de Pediatria do Curso de Graduação em Medicina da Faculdade Santa Maria - FSM - Cajazeiras, PB. https://orcid. org/0000-0003-0390-805X. E-mail: thaiseabreu@hotmail.com.

3. Doutora em Ciências da Saúde pela Faculdade de Medicina do ABC. Professora adjunta do Curso de Graduação em Medicina da Faculdade Santa Maria - FSM - Cajazeiras, PB. https://orcid.org/0000-0002-4751-2404. E-mail: ankilmar@hotmail.com.

4. Professora do Departamento de Clínica Médica do Curso de Graduação em Medicina da Faculdade Santa Maria - FSM - Cajazeiras, PB. https://orcid. org/0000-0003-1050-0239. E-mail: lucianamodesto@hotmail.com

Endereço para correspondência: Secretaria do curso de Graduação em Medicina da Faculdade Santa Maria. BR. 230 , Km 504 - Bairro Cristo Rei. Cajazeiras, PB. CEP: 58900-000.1 0974.E-mail: rd_ro@hotmail.com. 


\section{INTRODUÇ̃̃o}

$\mathrm{O}$ sono é um fenômeno vital, necessário à manutenção da homeostase corporal. O processo de dormir adequadamente tem funções biológicas fundamentais na consolidação de diversos processos como memória e restauração da energia corporal ${ }^{1,8}$. Alterações na qualidade do sono tem sido associadas à depressão, à ansiedade $\mathrm{e}^{2,3}$ e ao consumo excessivo de estimulantes cerebrais entre estudantes de medicina no Brasil ${ }^{4}$.

A qualidade do sono, tanto em aspectos subjetivos como em quantitativos, tem sido estudada entre estudantes de medicina $^{5-7}$. Os estudos nessa população tendem a demonstrar privação de sono, que está associada à elevada carga horária acadêmica e às tarefas em variadas atividades, o que acaba gerando impactos importantes na qualidade de vida do estudante. Estima-se que os prejuízos estejam relacionados à queda da produtividade, déficit cognitivo, desmotivação e prejuízo da saúde geral ${ }^{8}$.

A privação de sono interfere também nos processos reguladores do estresse. É interessante a observação do aumento dos níveis de cortisol em pessoas privados de sono. O que acontece é que a concentração sérica de cortisol é retroalimentada negativamente, ao longo do dia, com seus menores níveis no início do sono. O processo de privação de sono provoca alterações na regulação da retroalimentação negativa, o que provoca maiores níveis séricos na noite seguinte ${ }^{9}$

Entendendo a importância que esse prejuízo traz para a vida do universitário, ressalta-se a importância da avaliação da qualidade do sono entre estudantes de medicina. É para tanto que, na literatura, existem instrumentos de auto avaliação da qualidade do sono como o Pittsburgh Sleep Quality Index (PSQI, Índice de Qualidade do Sono de Pittsburgh) ${ }^{10}$ que é composto por 19 itens que abrangem desde a quantidade de horas de sono por noite ao uso de medicação para dormir, e a Escala de Sonolência Epworth $(\mathrm{ESE})^{11}$, que possui oito afirmações sobre a tendência à sonolência diurna em situações cotidianas, levando-se em conta o modo de vida do indivíduo nas últimas semanas.

Ambos os instrumentos utilizados na presente pesquisa foram validados e traduzidos para o português falado no Brasil e possuem elevada eficácia $(80 \%$ de especificidade e $68,8 \%$ de sensibilidade para o PSQI) ${ }^{12}$, tendo seu uso recomendado tanto para a prática clínica como também para a pesquisa científica. Desse modo, o objetivo do presente estudo foi avaliar a percepção da qualidade de sono e o grau de sonolência diurna excessiva entre estudantes de medicina, comparando as fases do curso, por meio da aplicação do PSQI e da ESE.

\section{MÉTODOS}

Trata-se de um estudo transversal e analítico, realizado através da aplicação de questionários individuais. Em relação à população e amostra, o estudo foi realizado com todos os estudantes do Curso de Graduação em Medicina de uma faculdade do alto sertão paraibano. A população foi composta pelos 341 alunos distribuídos nos 12 períodos de graduação em Medicina da instituição à época da aplicação dos questionários e não foi utilizado cálculo amostral, uma vez que a amostra foi representada pela população (excluindo-se apenas os que não aceitaram participar, os excluídos por critérios de exclusão e os que preencheram incorretamente o questionário).

Como critérios de inclusão, participaram do estudo todos os alunos devidamente matriculados no curso de Medicina da instituição que eram maiores de 18 anos que responderam de forma completa o PSQI e a ESE durante o período estabelecido e que concordaram com as condições do Termo de Consentimento Livre e Esclarecido (TCLE).

Os alunos que declinaram o convite de participação da pesquisa ou não preencheram os questionários corretamente, foram excluídos. A coleta de dados foi realizada no início do semestre letivo durante os meses de agosto e setembro de 2018, sendo que a aplicação ocorreu durante intervalo de aula ou imediatamente antes do seu início. O aplicador fez leitura prévia do TCLE, após o que os acadêmicos o assinaram. O presente estudo foi apreciado e aprovado pelo Comitê de Ética em Pesquisa em seres humanos da instituição proponente, sob o protocolo $\mathrm{n}^{\circ}$ 2.713.703/2018.

Utilizaram-se dois questionários autoaplicativos: o $\mathrm{PSQI}^{10}$ e a $\mathrm{ESE}^{11}$, traduzidos e validados para a língua portuguesa $^{12}$. O PSQI, utilizado para avaliar a qualidade subjetiva do sono, é composto por 19 itens agrupados em sete componentes, cada qual pontuado em uma escala de zero a três. Os componentes avaliados são: 1. A qualidade subjetiva do sono; 2. A latência do sono; 3. A duração do sono; 4. A eficiência habitual do sono; 5. Distúrbios relacionados ao sono; 6 . O uso de medicações para o sono; e 7. A disfunção diurna em relação ao ciclo vigília-sono.

Os escores dos sete componentes do são então somados para conferir uma pontuação global, a qual varia de 0 a 21. Pontuações de zero a cinco indicam boa qualidade do sono, maiores que ou iguais a 6 indicam qualidade ruim e acima de 10 indicam distúrbio do sono.

Em relação à ESE, trata-se de um questionário simples de 8 itens que são graduados de zero a três pontos, de acordo com a intensidade da sonolência. Cada item representa uma situação cotidiana habitual, sendo o objetivo analisar a chance que há de se cochilar em cada situação apresentada. Somam-se então os resultados obtidos nos 8 itens, sendo a pontuação tanto mais alta quanto maior for a chance de sonolência. O total de pontos possível é de 24 , considerando-se sonolência excessiva diurna um escore maior ou igual a 11 pontos. A seleção dos instrumentos desta pesquisa deveu-se ao fato de que essas são ferramentas de análise difundidas mundialmente e 
validadas para a avaliação de distúrbios do sono.

Os questionários aproveitados tiveram seus dados computados em tabelas com o uso do programa Microsoft Excel. Os dados coletados foram inseridos em banco de dados no programa de software Statistical Package for Social Sciences (SPSS), versão 20.0 para Windows, para posterior análise. Neste estudo, agruparam-se os anos para estudo seguindo os ciclos tradicionais do curso de medicina. A partir desse agrupamento, foi realizada a descrição dos resultados para o grupo geral e a comparação dos resultados entre os grupos nos três ciclos: Ciclo Básico (CB), com alunos no $1^{\circ}$ e $2^{\circ}$ anos de graduação; Ciclo Clínico (CC), com alunos no $3^{\circ}$ e $4^{\circ}$ anos; e Ciclo do Internato (CI), com alunos no $5^{\circ}$ e $6^{\circ}$ anos. Para a análise específica de significância estatística, foram realizados testes Quiquadrado e ANOVA. Neste estudo, valores de $\mathrm{p}<0,05$ foram considerados significativos.

\section{RESULTADOS}

Dos 341 alunos distribuídos nos 12 períodos de graduação em Medicina da instituição à época da aplicação dos questionários, aceitaram participar 279 acadêmicos, totalizando $81,81 \%$ do total de matriculados. Desta parcela, foram excluídos 45 por preenchimento incompleto e/ou indevido do questionário, resultando em uma amostra final de 234 participantes, o que representa por sua vez uma porcentagem final de $68,62 \%$ do total de alunos do curso sendo estudados. A média de idade foi de 23,4 anos com desvio padrão (DP) de 4,611. A Tabela 1 demonstra o perfil da amostra estudada, que foi dividida em ciclos.

$\mathrm{Na}$ amostra geral, 47,6\% dos participantes adquiriram entre 6 e 10 pontos, o que evidencia qualidade ruim do sono, enquanto $16,9 \%$ pontuou 11 ou mais, o que indica distúrbio do sono que deve ser investigado. Os resultados do PSQI foram analisados tanto na amostra geral (Tabela 2) quanto nos três grupos, individualmente, com seus componentes (Tabela 3 ).

No geral, a pontuação média no PSQI foi de 7,09 $(\mathrm{DP}=2,520)$. No grupo do $\mathrm{CB}$, a média foi de $7,15(\mathrm{DP}=$ 2,538), no CC foi de 7,04 (DP =2,788) e no CI foi de 6,89
$(\mathrm{DP}=2,622) . \mathrm{O}$-valor na análise comparativa do PSQI foi de 0,890 . Comparando os grupos vimos que $75,6 \%$ dos alunos do CB pontuaram 6 ou mais no PSQI, ou seja, possuem qualidade ruim ou distúrbio do sono, sendo essa taxa de $63,6 \%$ para o CC e de $68,4 \%$ para o CI. O valor do teste de Qui-quadrado de Pearson foi de 0,18.

Tabela 1. Distribuição da amostra pelo ciclo de graduação e por gênero

$\begin{array}{lcc}\text { Caracterização da amostra } & \text { Alunos; n (\%) } \\ \text { Grupos } & \text { Grupo CB } & 137(58,5) \\ & \text { Grupo CC } & 78(33,3) \\ & \text { Grupo CI } & 19(8,2) \\ \text { Gênero } & \text { Masculino } & 121(51,7) \\ & \text { Feminino } & 113(48,3)\end{array}$

Fonte: Dados do estudo; CB: Ciclo Básico; CC: Ciclo Clínico; CI: Ciclo do Internato

Tabela 2. Análise global final dos componentes do PSQI

\begin{tabular}{l|c|c}
\hline Qualidade do sono - PSQI & \% & N \\
\hline Boa (0 a 5 pontos) & 35,5 & 82 \\
\hline Ruim (6 a 10 pontos) & 47,6 & 110 \\
\hline Distúrbio do sono ( $\geq$ 11 pontos) & 16,9 & 39 \\
\hline Total Geral & 100,00 & 234 \\
\hline
\end{tabular}

Fonte: Dados do estudo;

O primeiro componente do PSQI avalia a percepção do estudante sobre a qualidade do próprio sono. Nesse sentido, um dado interessante encontrado foi o seguinte: subjetivamente, $65,9 \%$ dos participantes classificaram a qualidade do sono como muito boa ou boa e $34,1 \%$ como muito ruim ou ruim (Gráfico 1), o que nos leva a crer que os alunos superestimam a qualidade do próprio sono pois, de acordo com a Tabela 2, apenas 35,5\% dos alunos possuem qualidade boa do sono (pois pontuaram no máximo 5 no PSQI).

Gráfico 1. Avaliação subjetiva da qualidade do sono

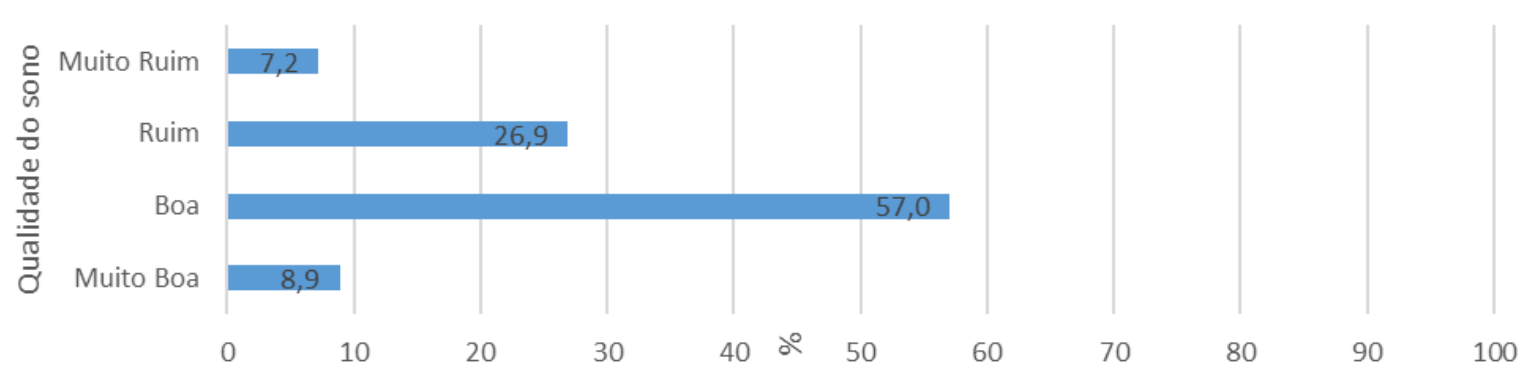

Fonte: Dados do estudo 
Silva RRP, et al. Qualidade do sono e sonolência excessiva entre estudantes de medicina.

Em relação à latência do sono, esta foi maior que 15 minutos para a maior parte dos entrevistados em cada grupo (Tabela 3). Considerando o terceiro componente avaliado, nosso estudo evidenciou que a média global de horas de sono por noite foi de 6,92 horas ( $\mathrm{DP}=1,239$ ). $58,6 \%$ dos entrevistados relataram dormir menos que 7 horas por noite. Estratificando em ciclos, tivemos que no $\mathrm{CB}$, no $\mathrm{CC}$ e no CI a média de horas de sono por noite é em torno de $7,04(\mathrm{DP}=1,278), 6,7(\mathrm{DP}=1,179)$ e $6,97(\mathrm{DP}=1,124)$, respectivamente. A comparação dos grupos entre si não revelou diferenças estatisticamente significantes ( $\mathrm{p}$-valor $=0,15)$. A eficiência habitual do sono foi maior que $85 \%$ para $67 \%$ dos participantes do CB, $64 \%$ do CI e $57 \%$ do CI.

Quanto ao uso de substâncias sedativas entre os acadêmicos, a maioria negou o uso $(79 \%, \mathrm{n}=185)$. Estratificando em ciclos, novamente a diferença não é significativa quando se considera o uso ao menos uma vez por semana (gira entre $20 \%$ e $25 \%$, aproximadamente, para os três grupos). Entre os que afirmaram usar tais medicações, foi considerável o número de alunos que os utilizavam mais de três vezes na semana, totalizando 14 alunos ( $6 \%$ do total).

Por fim, dos 7 componentes avaliados no PSQI, aquele que demonstrou maior relevância estatística $(\mathrm{p}=$ 0,003 ) foi o componente que avaliou a disfunção durante o dia. Neste componente, o entrevistado é convidado a responder com que frequência teve dificuldade para manter-se acordado durante atividades habituais ou manter o ânimo diário. O grupo que apresentou maiores taxas de disfunções severas foi o CC (22\%), contra $12 \%$ do $\mathrm{CB}$ e $5 \%$ do CI. Neste estudo, a maior parte da amostra $(53,9 \%)$ relatou ter dificuldade moderada ou severa para manter-se acordado durante o dia.

Tabela 3. Resultados dos componentes do PSQI em função dos ciclos e componentes individuais

\begin{tabular}{|c|c|c|c|c|c|c|}
\hline PSQI & $\underset{58,5 \%}{\text { Grupo }} \underset{5 B}{C(N}=137)$ & $\begin{array}{c}\text { Grupo CC } \\
(\mathbf{N}=78) \\
33,3 \%\end{array}$ & $\begin{array}{c}\text { Grupo CIC } \\
(\mathbf{N}=19) \\
8,2 \%\end{array}$ & n & $\%$ & \\
\hline \multicolumn{7}{|c|}{ Qualidade subjetiva do sono } \\
\hline Muito Boa & 12 & 7 & 2 & 21 & 8,9 & \multirow{4}{*}{$P=0,921$} \\
\hline Boa & 77 & 44 & 12 & 133 & 57,0 & \\
\hline Ruim & 38 & 20 & 5 & 63 & 26,9 & \\
\hline Muito Ruim & 10 & 7 & 0 & 17 & 7,2 & \\
\hline \multicolumn{7}{|c|}{ Latência do sono } \\
\hline$\leq 15 \min$ & 40 & 23 & 3 & 66 & 28,3 & \multirow{4}{*}{$P=\mathbf{0 , 5 2 0}$} \\
\hline 16 a $30 \mathrm{~min}$ & 64 & 30 & 9 & 103 & 44,0 & \\
\hline 31 a $60 \mathrm{~min}$ & 18 & 17 & 5 & 40 & 17,0 & \\
\hline $60 \mathrm{~min}$ & 15 & 8 & 2 & 25 & 10,7 & \\
\hline \multicolumn{7}{|c|}{ Duração do sono } \\
\hline$>7$ horas & 53 & 36 & 8 & 97 & 41,4 & \multirow{4}{*}{$P=0,648$} \\
\hline 6 a 7 horas & 65 & 34 & 8 & 107 & 45,7 & \\
\hline 5 a 6 horas & 13 & 4 & 3 & 20 & 8,5 & \\
\hline$<5$ horas & 6 & 4 & 0 & 10 & 4,4 & \\
\hline \multicolumn{7}{|c|}{ Eficiência habitual do sono } \\
\hline$>85 \%$ & 92 & 50 & 11 & 153 & 65,3 & \multirow{4}{*}{$P=0,740$} \\
\hline $75 \%$ a $84 \%$ & 33 & 22 & 5 & 60 & 25,7 & \\
\hline $65 \%$ a $74 \%$ & 8 & 2 & 2 & 12 & 5,1 & \\
\hline$<65 \%$ & 4 & 4 & 1 & 9 & 3,9 & \\
\hline \multicolumn{7}{|c|}{ Distúrbio do sono } \\
\hline $\mathbf{0}$ & 2 & 2 & 2 & 6 & 2,5 & \multirow{4}{*}{$\mathbf{P}=\mathbf{0 , 1 8 4}$} \\
\hline 1 a 9 & 94 & 61 & 15 & 170 & 72,6 & \\
\hline 10 a 18 & 39 & 13 & 2 & 54 & 23,1 & \\
\hline 19 a 27 & 2 & 2 & 0 & 4 & 1,8 & \\
\hline
\end{tabular}


Tabela 3. Resultados dos componentes do PSQI em função dos ciclos e componentes individuais

continuação

\begin{tabular}{|c|c|c|c|c|c|c|}
\hline PSQI & 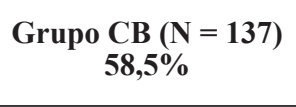 & $\begin{array}{c}\text { Grupo CC } \\
(\mathbf{N}=78) \\
33,3 \%\end{array}$ & $\begin{array}{c}\text { Grupo CIC } \\
(\mathbf{N}=19) \\
\mathbf{8 , 2 \%}\end{array}$ & n & $\%$ & \\
\hline \multicolumn{7}{|c|}{ Uso de medicamentos para dormir } \\
\hline Nenhuma vez por semana & 108 & 63 & 14 & 185 & 79,0 & \multirow{4}{*}{$P=0,168$} \\
\hline Menos de uma vez /semana & 12 & 5 & 5 & 22 & 9,5 & \\
\hline Uma ou duas vezes/semana & 8 & 5 & 0 & 13 & 5,5 & \\
\hline Mais de três vezes/semana & 9 & 5 & 0 & 14 & 6,0 & \\
\hline \multicolumn{7}{|c|}{ Disfunção durante o dia } \\
\hline Nenhuma - 0 & 17 & 3 & 2 & 22 & 9,4 & \multirow{4}{*}{$\mathbf{P}=\mathbf{0 , 0 0 3}$} \\
\hline Leve - 1 a 2 & 45 & 32 & 9 & 86 & 36,7 & \\
\hline Moderada - 3 a 4 & 58 & 26 & 7 & 91 & 38,9 & \\
\hline Severa - 5 a 7 & 17 & 17 & 1 & 35 & 15,0 & \\
\hline
\end{tabular}

Fonte: Dados do estudo; CB: Ciclo Básico; CC: Ciclo Clínico; e CI: ciclo do Internato

Em relação à Escala de Sonolência de Epworth (ESE), a análise dos dados obtidos demonstrou que 55,5\% $(\mathrm{n}=130)$ dos estudantes pontuaram 11 ou mais, ou seja, têm sonolência diurna excessiva que deve ser investigada (Tabela 4). Comparando os ciclos, concluímos que 45\% do grupo CB apresentou sonolência diurna excessiva, enquanto esse valor foi de $48 \%$ para o CC e de $26 \%$ para o CIC.

Não foram encontradas diferenças estatisticamente significantes em relação à ESE e ao sexo $(p=0,120)$, ao ciclo básico $(\mathrm{p}=0,295)$, ao ciclo clínico $(\mathrm{p}=0,704)$ ou ao ciclo do internato $(\mathrm{p}=0,270)$, o que demonstra mais uma vez a homogeneidade dos grupos com relação aos parâmetros utilizados.

Tabela 4. Sonolência diurna excessiva de acordo com a escala de sonolência de Epworth

\begin{tabular}{llcc}
\hline Pontuação & Significado & $\mathbf{\%}$ & $\mathbf{n}$ \\
\hline $\mathbf{0 - 6}$ & Normal & 19,65 & 46 \\
\hline $\mathbf{7}$ a $\mathbf{1 0}$ & Limítrofe & 23,07 & 54 \\
\hline $\mathbf{1 1}$ a $\mathbf{1 5}$ & Sonolência Diurna Excessiva & 42,3 & 99 \\
\hline $\mathbf{1 6}$ a $\mathbf{1 9}$ & Sonolência Diurna Excessiva & 12,39 & 29 \\
\hline $\mathbf{2 0}$ ou mais & Sonolência Diurna Excessiva & 0,85 & 2 \\
\hline Total & & 100,0 & 234 \\
\hline Fonte: Dados do estudo & & & \\
\hline
\end{tabular}

\section{DISCUSSÃO}

A qualidade do sono entre estudantes de medicina é um tema que vem sendo explorado tanto no Brasil quanto mundo afora, principalmente, levando em consideração as repercussões silenciosas na qualidade de vida desta população. Foram entrevistados neste estudo, com aproveitamento, $68,62 \%$ de todos os graduandos em Medicina da instituição, o que se aproxima da média avaliada em outros estudos nesse grupo ${ }^{5,8}$

A amostra global deste estudo apresentou uma média de horas de sono por noite de $6,92 \mathrm{~h}$, taxa menor que a média da população adulta em geral (sete a nove horas) $)^{13}$, porém maior que a encontrada em outras pesquisas com a mesma população, com médias de $6,13 \mathrm{~h}$ em Goiás ${ }^{6} 6,8 \mathrm{~h}$ em São Paulo ${ }^{14}$ e 6,48h entre estudantes de Medicina da Argentina $^{15}$, sendo importante salientar que não houve diferença significativa entre os grupos analisados $(\mathrm{CB}, \mathrm{CC}$ e CI) no quesito horas de sono por noite.

Ao se analisar a qualidade do sono à luz dos instrumentos utilizados, este estudo apontou que $64,5 \%$ da $\operatorname{amostra}(n=149)$ adquiriu mais que 5 pontos no PSQI, taxa discrepante à encontrada entre acadêmicos da Universidade Federal do Acre $(76,3 \%)^{8}$, de Botucatu - SP $(87,1 \%)^{5}$ e semelhante a de estudo realizado no Sul Catarinense, onde $64,7 \%$ dos estudantes de Medicina apresentaram este padrão ${ }^{16}$. No nosso estudo, a análise comparativa entre os grupos utilizando o PSQI não revelou diferenças estatísticas significantes, o que evidencia uma homogeneidade entre os grupos avaliados. 
Um dado interessante foi o de que os alunos superestimam a qualidade do próprio sono. Quando entrevistados, apenas $34,1 \%$ afirmaram achar a qualidade do sono ruim ou muito ruim, quando na verdade $64,5 \%$ tem qualidade ruim do sono ou distúrbio deste, pois tiveram mais que 5 pontos no PSQI. Isso demonstra a discordância entre a autopercepção da qualidade de sono e a realidade. É de se supor que esta problematização seja prejudicada pelo fato de que não se reconhece a existência de um problema, quando sabidamente a qualidade do sono entre estudantes de medicina é pior ${ }^{17}$

Sem dúvida, um dos dados mais inesperados e preocupantes do estudo foi a significativa parcela de estudantes de Medicina que relataram usar medicação sedativo-hipnótica para dormir ao menos uma vez por semana durante o mês que antecedeu a aplicação da pesquisa. Em nosso estudo este índice chegou a 21\%, taxa superior à encontrada na literatura, onde essa taxa variou entre índices como: $7,01 \%^{14} ; 8,6 \%{ }^{5} \mathrm{e} 9,0 \%{ }^{7}$. A preocupação atual com o incremento do uso de drogas sedativas pelos estudantes se dá pelo risco de toxicodependência e pelos efeitos colaterais advindos do abuso de tais substâncias.

Sonolência diurna excessiva foi encontrada em $55,55 \%$ da amostra estudada. É interessante notar que essa taxa se aproxima da encontrada em outros estudos: $49,8 \%{ }^{18}$ e $47,1 \%{ }^{20}$. A causa mais comum de sonolência excessiva diurna é a privação crônica de sono. A quantidade necessária de sono tem variações individuais e parece ser determinada geneticamente ${ }^{19}$.

Entre as limitações deste estudo cabe ressaltar que variáveis capazes de influenciar na ocorrência de distúrbios do sono (como preparação para processos seletivos, conflitos familiares, padrão alimentar, atividades físicas) não foram analisadas. Todavia, é importante entender a dimensão da problemática analisada. Padrão ruim de qualidade do sono entre estudantes de medicina deve ser uma preocupação constante para todos os envolvidos, levando em consideração os prejuízos acarretados, como piora do desempenho acadêmico e da atenção ${ }^{21,22} \mathrm{e} o$ aumento da prevalência de transtornos mentais comuns como dificuldade de concentração e na tomada de decisões, irritabilidade e fadiga ${ }^{23}$.
Certamente é relevante discutir, para além dos parâmetros técnicos, os parâmetros subjetivos dos pontos abordados. Como podemos analisar os dados que foram fornecidos por este estudo? Como podemos aplicá-los de forma prática no cotidiano do estudante de medicina? Como podemos definir até que ponto mais tempo acordado significa maior produção? Fatidicamente, um dos objetivos desta produção foi fornecer subsídios para alimentar essa discussão;

Entende-se que há sim déficit na qualidade do sono do estudante de medicina. Não só nosso estudo revelou isso, como também vários outros acima apontados. Frente a isso, e sabendo dos impactos que decorrem da situação, é necessário propor mudanças. Não se pode aceitar sacrificar a saúde mental do estudante pela possibilidade de uma maior produção acadêmica; Ora, é inclusive paradoxo pregar a saúde e o bem-estar, estar na graduação de um curso que promove saúde, e por trás sacrificar este bem por uma expectativa de maior produção acadêmica, que muitas vezes nem é alcançada, tendo em vista a queda de produção de um estudante mentalmente exausto.

\section{CONSIDERAÇÕES FINAIS}

Conclui-se a partir deste estudo que o grupo de estudantes avaliado possui em sua maioria um padrão ruim de qualidade sono, o que é preocupante. Os resultados obtidos indicam que muitos estudantes referem além de sonolência diurna excessiva, uso abusivo de medicação para dormir. O presente estudo chama atenção para a necessidade de se levar em consideração o sono do estudante como uma ferramenta para a melhorar a compreensão do bem-estar psicológico e da boa qualidade de vida dos acadêmicos.

Ademais, aponta-se para a pertinência da importância do investimento de esforços para a correção dessa tendência. Fazem-se necessárias medidas de promoção de saúde entre a população de estudantes do ensino superior, especialmente aqueles que sofrem com uma carga horária elevada e uma agenda por vezes difícil de cumprir, como estudantes de medicina, com propostas de modificação dos comportamentos de saúde adotados para melhorar, por exemplo, a higiene de sono.

Suporte financeiro: Manuscrito produzido com recursos próprios.

Agradecimentos: Primeiramente à Deus, por nos permitir chegar até aqui; Agradecemos ainda, imensamente, à Faculdade Santa Maria pelo apoio e pelo suporte no desenvolvimento técnico deste estudo.

Participação dos autores: Rodrigo Rufino Pereira Silva: Participou na elaboração do projeto, coleta de dados, análise dos dados, discussão dos achados, redação e edição do artigo, submissão do artigo; Thaise de Abreu Brasileiro Sarmento: Contribuiu com a concepção e com orientações na elaboração do projeto, análise dos dados, discussão dos achados; Ankilma do Nascimento Andrade Feitosa: Acompanhou a confecção e o desenvolvimento da pesquisa, além do auxílio na edição do texto e revisão final; Luciana Modesto de Brito: Acompanhou a pesquisa com edição e delineamento do texto, revisão final e submissão do artigo. 


\section{REFERÊNCIAS}

1. Lewis SR, Pritchard MW, Schofield-Robinson OJ, Alderson $\mathrm{P}$, Smith AF. Melatonin for the promotion of sleep in adults in the intensive care unit. Cochrane Database of Systematic Rev. 2018;5:CD012455. doi: http:// dx.doi. org/10.1002/14651858.CD012455.pub2.

2. Leao AM, Gomes IP, Ferreira MJM, Cavalcanti LPG. Prevalência e fatores associados à depressão e ansiedade entre estudantes universitários da área da saúde de um grande centro urbano do Nordeste do Brasil. Rev Bras Educ Med. 2018;42(4):55-65. doi: http://dx.doi. org/10.1590/1981-52712015v42n4rb20180092.

3. Santa ND, Cantilino A. Suicídio entre médicos e estudantes de medicina: revisão de literatura. Rev Bras Educ Med. 2016;40(4):772-80. doi: http://dx.doi.org/10.1590/1981$52712015 \mathrm{v} 40 \mathrm{n} 4 \mathrm{e} 00262015$.

4. Morgan HL, Petry AF, Licks PAK, Ballester AO, Teixeira $\mathrm{KN}$, Dumith SC. Consumo de estimulantes cerebrais por estudantes de medicina de uma universidade do extremo Sul do Brasil: prevalência, motivação e efeitos percebidos. Rev Bras Educ Med. 2017;41(1):102-9. doi: http://dx.doi. org/10.1590/1981-52712015v41n1rb20160035.

5. Corrêa CC, Oliveira FK, Pizzamiglio DS, Ortolan EVP, Weber SAT. Qualidade de sono em estudantes de medicina: comparação das diferentes fases do curso. J Bras Pneumol. 2017;43(4):285-9. doi: http://dx.doi.org/10.1590/S180637562016000000178 .

6. Cardoso HC, Bueno FC, Mata JC, Alves AP, Jochims I, Vaz Filho IH, et al. Assessing quality of sleep in medical students. Rev Bras Educ Med. 2009;33(3):349-55. https:// dx.doi.org/10.1590/S0100-55022009000300005.

7. Alsaggaf MA, Wali SO, Merdad RA, Merdad LA. Sleep quantity, quality, and insomnia symptoms of medical students during clinical years. Saudi Med J. 2016;37(2):17382. doi: http://dx.doi.org/10.15537/smj.2016.2.14288.

8. Ribeiro CR, Oliveira SM, Silva YM. The impact of sleep quality in medical. Rev Soc Bras Clin Med. 2014;12(1):814. [Cited 2019 July 01]. Available from: http://files.bvs. br/upload/S/1679-1010/2014/v12n1/a4027.pdf.

9. Besedovsky L, Lange T, Born J. Sleep and immune function. Pflugers Arch. 2012;463(1):121-37. doi: https://dx.doi. org/10.1007/s00424-011-1044-0.

10. Buysse DJ, Reynolds CF 3rd, Monk TH, Berman SR, Kupfer DJ. The Pittsburgh Sleep Quality Index: a new instrument for psychiatric practice and research. Psychiatry Res. 1989;28(2):193-213. doi: https://doi.org/10.1016/01651781(89)90047-4.

11. Johns MW. A new method for measuring daytime sleepiness: the Epworth sleepiness scale. Sleep. 1991; 14: 540-545. doi: https://doi.org/10.1093/sleep/14.6.540.

12. Bertolazi AN. Tradução, adaptação cultural e validação de dois instrumentos de avaliação do sono: escala de sonolência de Epworth e índice de qualidade de sono de Pittsburgh [Dissertação]. Porto Alegre: Universidade Federal do Rio Grande do Sul; 2008. Disponível em: http://www. lume.ufrgs.br/ bitstream/handle/10183/14041/000653543. pdf? sequence $=1$.

13. Poyares D, Tufik S. I Consenso Brasileiro de Insônia: introdução. Hypnos: rev sono. São Paulo: Sociedade Brasileira de Sono; 2003. p.5 [citado 01 jul. 2019]. Disponível em: http://jararaca.ufsm.br/websites/lan/ download/Consensos/insonia.pdf.

14. Moraes CAT, Edelmuth DGL, Novo NF, Hübner CVK. Qualidade de sono em estudantes de medicina do método de aprendizado baseado em problemas. Medicina (Ribeirão Preto). 2013;46(4):389-97. doi: https://dx.doi. org/10.11606/issn.2176-7262.v46i4p389-397.

15. Fontana SA, Raimondi W, Rizzo ML. Quality of sleep and selective attention in university students: descriptive crosssectional study. Medwave. 2014;14(8):e6015. doi: https:// dx.doi.org/10.5867/medwave.2014.08.6015.

16. Fonseca ALP, Zeni LB, Flügel NT, Sakae TM, Remor KVT. Estudo comparativo sobre qualidade do sono entre universitários de uma instituição de ensino do sul catarinense. Arq Catarin Med. 2015;44(4):21-33. Disponível em: http://www.acm.org.br/acm/seer/index.php/ arquivos/article/view/45.

17. Carvalho TMCS, Silva Junior II, Siqueira PPS, Almeida JO, Soares AF, Lima AMJ. Qualidade do sono e sonolência diurna entre estudantes universitários de diferentes áreas. Rev Neurocienc 2013;21(3):383-7. doi: https://dx.doi. org10.4181/RNC.2013.21.854.5p.

18. Machado-Duque ME, JEE, Machado-Alba JE. Somnolencia diurna excesiva, calidad del sueño y bajo desempeño académico en estudiantes de medicina. Rev Colomb Psiquiatr. 2015;44(3):137-42. doi: http://dx.doi. org/10.10167j.rcp.2015.04.002.

19. Frasson MZ, Monteiro LF, Corrêa JM, Wrzesinski A. Avaliação da qualidade do sono e fatores associados em estudantes de medicina. Arq Catarin Med. 2014;43(4):347. Disponível em: http://www.acm.org.br/revista/pdf/ artigos/1311.pdf.

20. Tufik S. Medicina e biologia do sono. Barueri (SP): Manole; 2008.

21. Pérez-Olmos I, Ibáñez-Pinilla M. Night shifts, sleep deprivation, and attention performance in medical students. Int J Med Educ. 2014;5:56-62. doi: http://dx.doi. org/10.5116/ijme.531a.f2c9.

22. Mirghani HO, Mohammed OS, Almurtadha YM, Ahmed MS. Good sleep quality is associated with better academic performance among Sudanese medical students. BMC Res Notes. 2015;8:706. doi: http://dx.doi.org/10.1186/s13104015-1712-9.

23. Ferreira CMG, Kluthcovsky ACGC, Cordeiro TMG. Prevalência de transtornos mentais comuns e fatores associados em estudantes de medicina: um estudo comparativo. Rev Bras Educ Med. 2016;40(2):268-77. doi: http://dx.doi.org/10.1590/1981-52712015v40n2e02812014.

Submetido: 05.07.2019

Aceito: 13.07.2020 